\title{
Performance of different silicon materials for the upgraded CMS tracker
}

\author{
Teppo Mäenpää*† \\ Helsinki Institute of Physics \\ E-mail: teppo.maenpaa@cern.ch
}

The proposed LHC luminosity upgrade (HL-LHC) would push the operating conditions of the CMS tracking system beyond the design limits, both in terms of radiation dose and channel occupancy. A comparison of various silicon materials and processing types has been launched in the framework of the Compact Muon Solenoid (CMS) experiment to find the best suited material for a future strip tracker. Detectors made of Float Zone and Magnetic Czochralski silicon, both $\mathrm{n}$-on-p and p-on-n types, have been produced. Two different isolation techniques, $\mathrm{p}$-stop and pspray, have been tested for the p-type sensors. Both $200 \mu \mathrm{m}$ and $320 \mu \mathrm{m}$ thick detectors have been irradiated with both protons and neutrons to the fluence foreseen at a radial position of $20 \mathrm{~cm}$ in the upgraded CMS experiment.

The tested Multi-geometry Silicon Strip Detectors (MSSD) contain 12 regions, where the region pitches range from 70 to $240 \mu \mathrm{m}$, and strip width/pitch (w/p) ratios from 0.1 to 0.3 . This article is based on test beam data for approximately 300 combinations of detector material, polarity, isolation type, thickness, pitch, and w/p. The test structures were measured using proton, muon, and pion beams, with beam energies between 15 and $300 \mathrm{GeV}$. The detectors were mounted perpendicular to the beam inside a cold box. Reference planes fore and aft of Detectors Under Test (DUT) were providing high-quality tracks, with a positional uncertainty of $6 \mu \mathrm{m}$.

The results show smaller than expected differences between the performance of the material types. After irradiation, the performance of thin detectors was comparable to or even exceeded the performance of the thicker detectors.

11th International Conference on Large Scale Applications and Radiation Hardness of Semiconductor Detectors

3-5 July 2013

Florence, Italy

\footnotetext{
* Speaker.

†n behalf of the CMS Tracker collaboration.
} 


\section{Introduction}

The proposed Large Hadron Collider (LHC) luminosity upgrade will affect the operation of the ongoing LHC experiments. Track density will increase ten-fold and radiation damage of the detectors will increase correspondingly. Radiation damage will reduce the amount of collected charge and increase the amount of leakage current and shot noise. Both these lower the signalto-noise ratio of the detectors and therefore increase the amount of fake clusters and lead to a reduction in the efficiency for reconstructing real hits. These effects will make particle tracking more difficult.

The Compact Muon Solenoid (CMS) experiment has started testing silicon detectors of various properties, described briefly below and in detail in References [1] and [2], to find the best suited material for a future CMS tracker. The detectors were irradiated to a combined (protons plus neutrons) fluence of $1.5 \times 10^{15} 1 \mathrm{MeV} \mathrm{n} \mathrm{n}_{\mathrm{eq}} / \mathrm{cm}^{2}$, and their tracking performance was measured in test beams before and after irradiation. The irradiation level corresponds to the integrated fluence expected at the end of HL-LHC [1] in the strip layers closest to the beam spot ( $20 \mathrm{~cm}$ radius).

\section{MSSD Detectors}

The Multi-geometry Silicon Strip Detectors (MSSD), manufactured by Hamamatsu Photonics K.K., comprise twelve regions, with 32 strips on each region (table 1). The strips are all $3 \mathrm{~cm}$ long. The nominal active thicknesses of the detectors were $200 \mu \mathrm{m}$ and $320 \mu \mathrm{m}$. Active thicknesses measured using a capacitance-vs-voltage method were $190 \mu \mathrm{m}, 220 \mu \mathrm{m}$ (deep diffusion, see below), and $300 \mu \mathrm{m}$.

Figure 1 shows the labeling scheme for the MSSD modules. Two types of material were used: Magnetic Czochralski (MCZ) and oxygenated Float Zone (FZ and FTH) silicon. All Czochralski detectors were physically thinned to $200 \mu \mathrm{m}$. Some Float Zone detectors were also physically thinned (FTH), while others were effectively thinned (active volume reduced) through a deep diffusion process, or kept at $320 \mu \mathrm{m}$ (FZ). Some of the wafers were p-on-n type (denoted as $\mathrm{N}$ in the label), while others were n-on-p. The n-on-p detectors utilized either the p-stop $(\mathrm{P})$ or the $\mathrm{p}$-spray (Y) method to isolate the strips. The other fields in the labels shown in Fig. 1 are used to identify the modules.

\section{Beam Tests}

The MSSD detectors were bonded to APV25 chips [3], which are used in the current CMS silicon strip tracker, prior to testing. The sensors and hybrids were attached to aluminum frames to form modules [4].

The detectors were tested in a Silicon Beam Telescope ( $\mathrm{SiBT}$ ) described in [5]. The temperature of the beam telescope enclosure was kept at $-20^{\circ} \mathrm{C}$ when irradiated detectors were measured, and at $+15^{\circ} \mathrm{C}$ otherwise. Sensor temperatures were not directly measured. Four beam tests have been carried out: one at the Fermilab MTEST, three at the CERN H2 beam lines.

The MSSD detector size is $66 \times 32 \mathrm{~mm}^{2}$, while the active area of the overlapped $4+4 \mathrm{SiBT}$ reference planes is $\sim 38 \times 38 \mathrm{~mm}^{2}$ [5]. The MSSD detectors were mounted in a $45^{\circ}$ orientation 


\begin{tabular}{l|cccccccccccc} 
region & 1 & 2 & 3 & 4 & 5 & 6 & 7 & 8 & 9 & 10 & 11 & 12 \\
& & & & & & & & & & & & \\
pitch & 120 & 240 & 80 & 70 & 120 & 240 & 80 & 70 & 120 & 240 & 80 & 70 \\
w/p [\%] & 13.3 & 14.2 & 12.5 & 12.1 & 23.3 & 24.2 & 22.5 & 22.1 & 33.3 & 34.2 & 32.5 & 32.1
\end{tabular}

Table 1: MSSD detector regions. Strip width in the width/pitch ratio refers to the silicon implant.

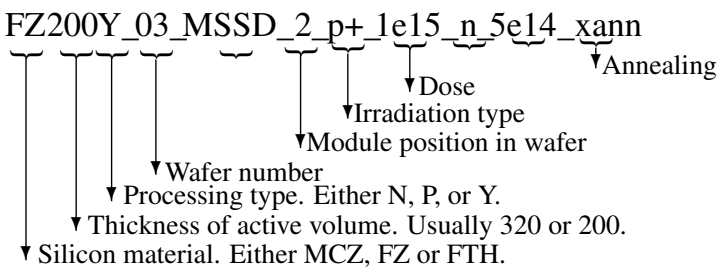

Figure 1: MSSD module names. If the irradiation type is omitted, then the quoted fluence is the total, in $1 \mathrm{MeV} \mathrm{n}$ eq $/ \mathrm{cm}^{2}$. If two module names differ only by irradiation or annealing field, then a single module has been tested both before and after irradiation.

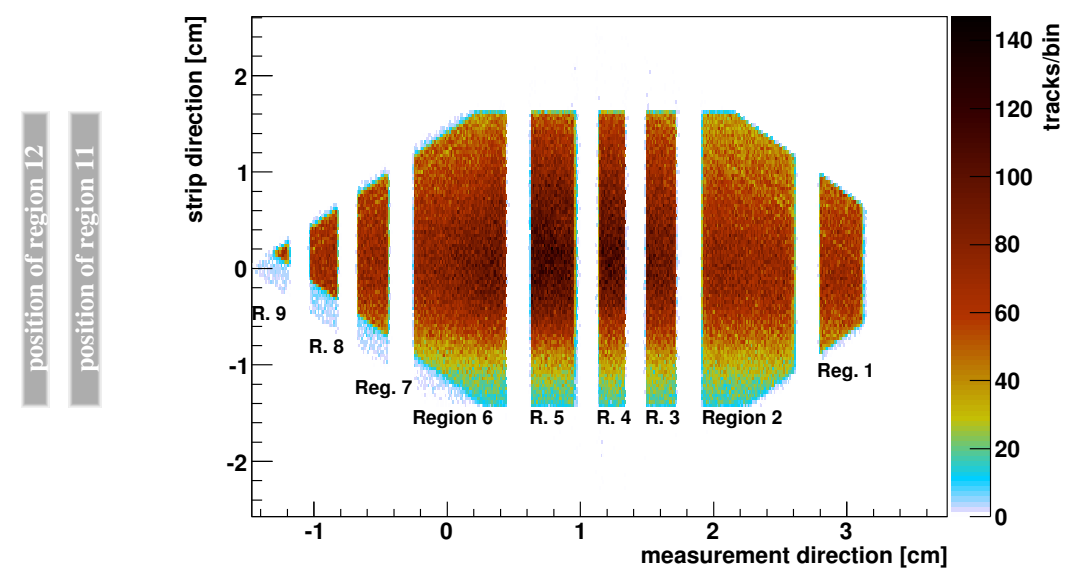

Figure 2: Positional distribution of tracks detected by a non-irradiated MSSD detector. The MSSD regions appear as vertical bars in this histogram. Diagonal contours are the SiBT reference plane boundaries. In this run, all regions with $\mathrm{w} / \mathrm{p} \sim 0.1$ have large statistics. Data from regions with $\mathrm{w} / \mathrm{p} \sim 0.2 \mathrm{might}$ be usable as well, while data from regions with $w / p \sim 0.3$ are unusable. The edge strips tend to have high noise values, and therefore they appear to find few tracks also when the particle did not pass through the active volume of the strip.

with respect to the reference detectors. This was necessary for mechanical reasons. This mounting increased the number of regions that were at least partially in the beam during data acquisition, at the expense of a slightly higher track position uncertainty: $6 \mu \mathrm{m}$ instead of $<4 \mu \mathrm{m}$. Figure 2 illustrates the geometry.

\section{Analysis}

The measurement data were analyzed within the CMS software framework, as described in [6]. A track-induced clustering (TIC) analysis flow, described in [7], was used to analyze the charge collection and noise properties, for the reasons described in Ref. [7]. For other results, the Three 


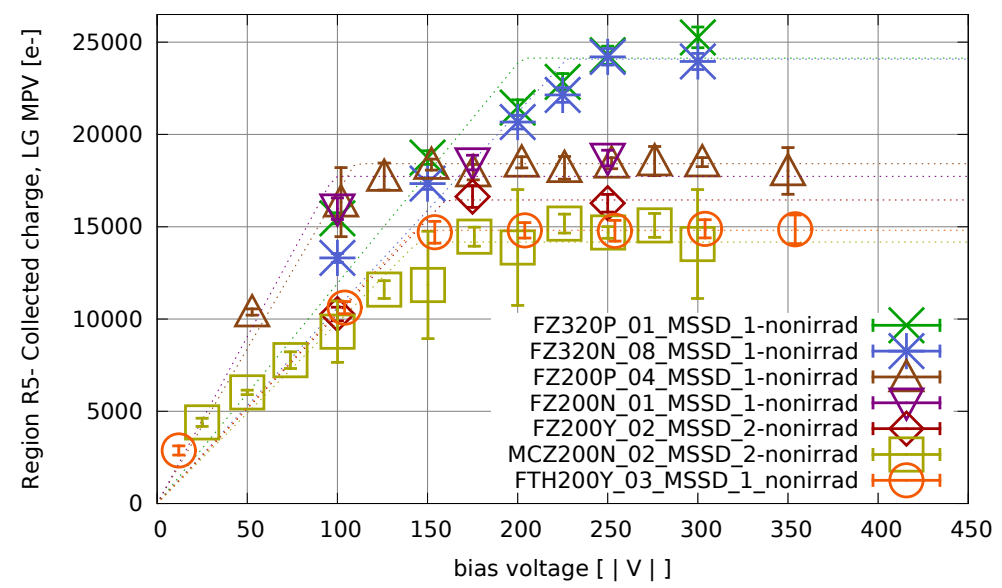

Figure 3: Charge collection of unirradiated detectors as a function of bias voltage. Most probable values (MPV) of convoluted Landau-Gaussian function (LG) fitted to the distribution of collected charges are shown.

Threshold Algorithm [8] was used with signal-to-noise ratio (SNR) thresholds of 3.5, 2.0, and 4.5 for seed, channel, and cluster, respectively The edge strips of each MSSD region were omitted, both from the common mode noise determination and from tracking, as they tended to have high noise values.

Reference data were generated using reference detectors only, with one exception: knowledge of the Device Under Test (DUT) active areas was used to discard ambiguous events as required by TIC. After reference data construction, each remaining event contained a single, well-measured reference track.

Deposited charge in the SiBT system is recorded in analog-to-digital (ADC) units. To compensate for variations in the expected charge due to beam type and beam energy, the most probable values of reference detector signal responses were set to be 23200 electrons (corresponding to $80 \mathrm{e}-/ \mu \mathrm{m})$, and the signals from the DUT detectors were converted from ADC units to electrons using the same factor.

\section{Results}

The charge collection of most detectors before irradiation scales with the thickness of the detector, as shown in Figure 3. Detectors thinned with deep diffusion collect more charge than predicted by the nominal active thickness of those detectors. After irradiation, the differences had disappeared (Fig. 4 upper left plot).

The differences between various materials are small (Fig.4). Detectors made of n-type and p-type silicon have very similar performances. Detectors made of Magnetic Czochralski material collect slightly more charge than those made of Float Zone material. There are small variations across the regions (Fig. 4), but the verdict remains the same. The fact that the post-irradiation charge collection efficiencies of the FZ and MCz detectors were similar could be explained by the elevated oxygen concentrations measured in the Float Zone detectors. 

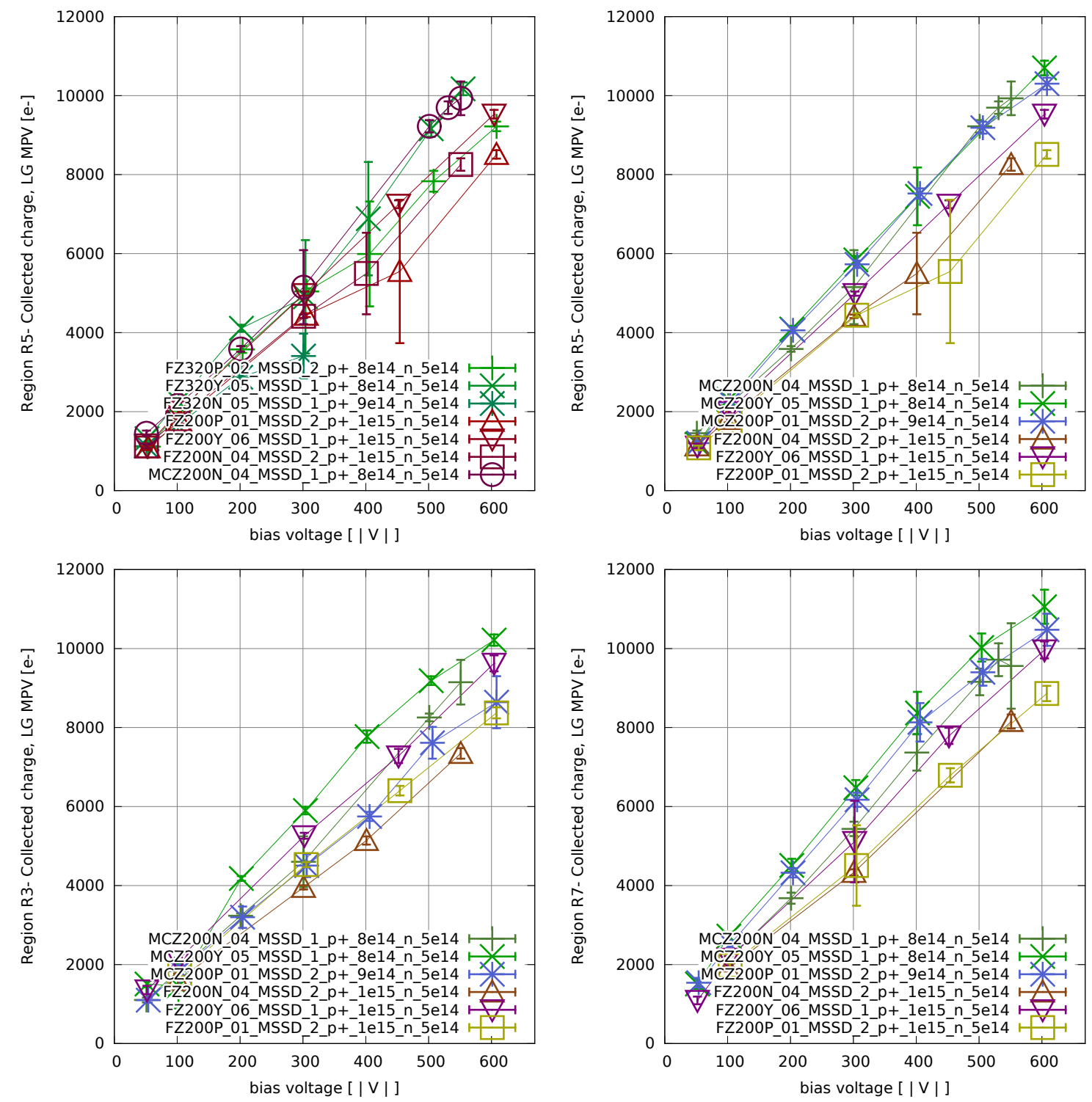

Figure 4: Charge collection of irradiated detectors as a function of bias voltage. Upper left plot compares the detector thicknesses, while the others compare detectors with a similar thickness to each other. Most probable values (MPV) of convoluted Landau-Gaussian function (LG) fitted to the distribution of collected charges are shown for regions $3(\mathrm{p}=80 \mu \mathrm{m}), 5(\mathrm{p}=120 \mu \mathrm{m})$, and $7(\mathrm{p}=80 \mu \mathrm{m})$. The lines merely join the data points.

Many detectors showed evidence of non-Gaussian noise. The effect varies from detector to detector and region to region; illustrative plots are shown in Figure 5. Because of the large search space and small number of samples, it is not possible to draw firm conclusions regarding the effect. However, non-Gaussian behavior did appear more frequently in n-type detectors than in p-type detectors. In most cases, the hit efficiencies of the n-type detectors are below those measured in p-type silicon, as shown in Figure 6.

The collected charge depends on the distance of the reference particle from the closest strip 

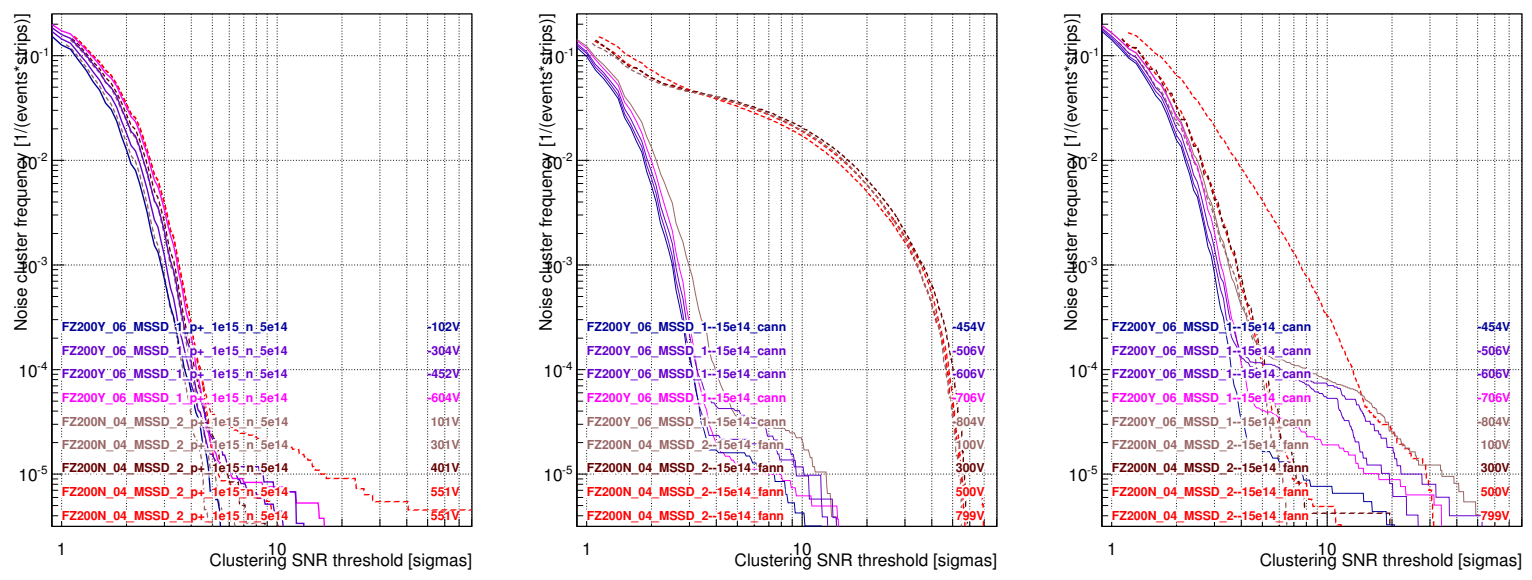

Figure 5: Each example plot shows the data of two detectors, at various bias voltages. The abscissa shows a clustering signal over noise ratio threshold, and the ordinate shows the probability of a strip producing a noise cluster. Data from n-type detectors are shown with dashed lines. A fixed value of noise in ADCs was used for all detectors while making these plots. The left and the center plots show region 11 data, the right plot shows region 3 of the same detectors. Data in the left plot was acquired prior annealing, data at center and right plots after annealing.

(Fig. 7). In the case of irradiated detectors and large pitch, the charge collected from far-strip tracks was less than $50 \%$ of the near-strip track induced charge, despite a higher bias voltage and a better overall charge collection as shown in Figure 7.

\section{Conclusions}

The performance of $200 \mu \mathrm{m}$ thick detectors was similar to or exceeded the performance of $320 \mu \mathrm{m}$ thick detectors, both in terms of charge collection and detector efficiency. Use of thin detectors is also beneficial for the tracker material budget.

It is generally known $[9,10,11]$ that the p-type detectors show higher charge collection efficiencies than n-type detectors after irradiation. The results of this study show that detector thinning improves the charge collection performance of irradiated n-type sensors, and significantly minimizes the tracking performance differences between n-type and p-type materials. The test beam results do not unambiguously point to one silicon type as the preferred choice, but rather suggest that there are a number of possibilities. So other considerations, such as the relative cost and ease of manufacturing, should be taken into account.

\section{References}

[1] R. D’Alessandro et al., Silicon Sensor and Detector Developments for the CMS Tracker Upgrade, PoS(EPS-HEP2011)201

[2] M. Bernard-Schwarz et al., Future silicon sensors for the CMS Tracker Upgrade, Nucl. Instr. \& Meth. A, vol. 699 (2013) 89-92 

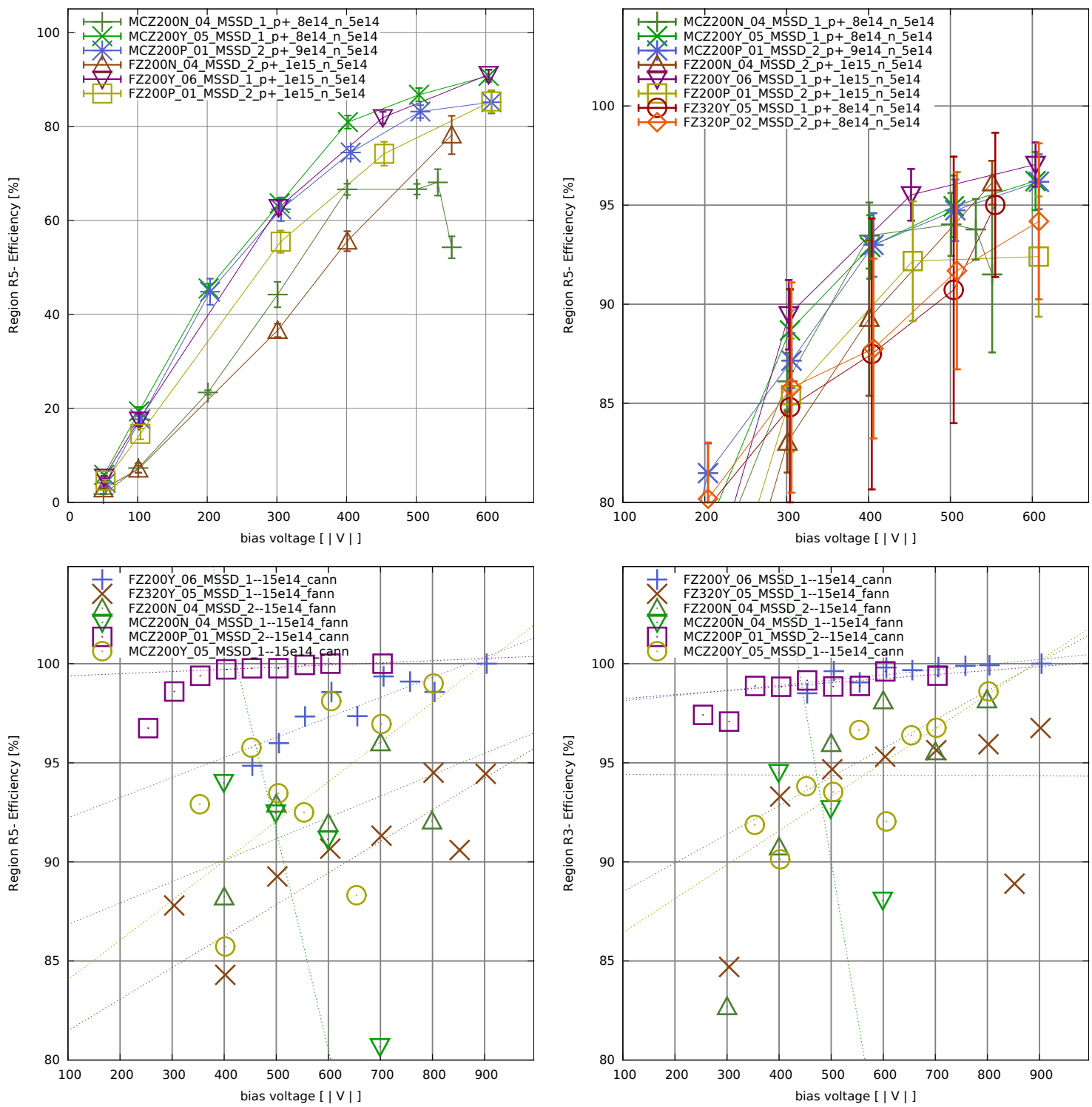

Figure 6: Efficiency of irradiated detectors as a function of bias voltage. The data points in the upper right plot are calculated using a fixed cluster threshold of 4.5 as described in text. Data points in the other plots are calculated by tuning the thresholds separately for each data point. Upper plots depict region $5(\mathrm{p}=120 \mu \mathrm{m})$ before annealing, while the lower plots show post-annealing data for regions 5 and $3(\mathrm{p}=80 \mu \mathrm{m})$. Detector efficiency varied significantly between runs in the latest datasets. Error bars were omitted from those plots for clarity.

[3] M.Raymond et al., The CMS Tracker APV25 $0.25 \mu \mathrm{m}$ CMOS Readout Chip, in proceedings of The 6th workshop on electronics for LHC experiments, Krakow, Poland, Sept. 2000

[4] G. Auzinger et al., Analysis of testbeam data of irradiated silicon prototype sensors for the CMS tracker upgrade, Nucl. Instr. \& Meth. A, in press.

[5] T. Mäenpää et al., Silicon Beam Telescope for LHC upgrade tests, Nucl. Instr. \& Meth. A, vol. 593 (2008) 523-9 

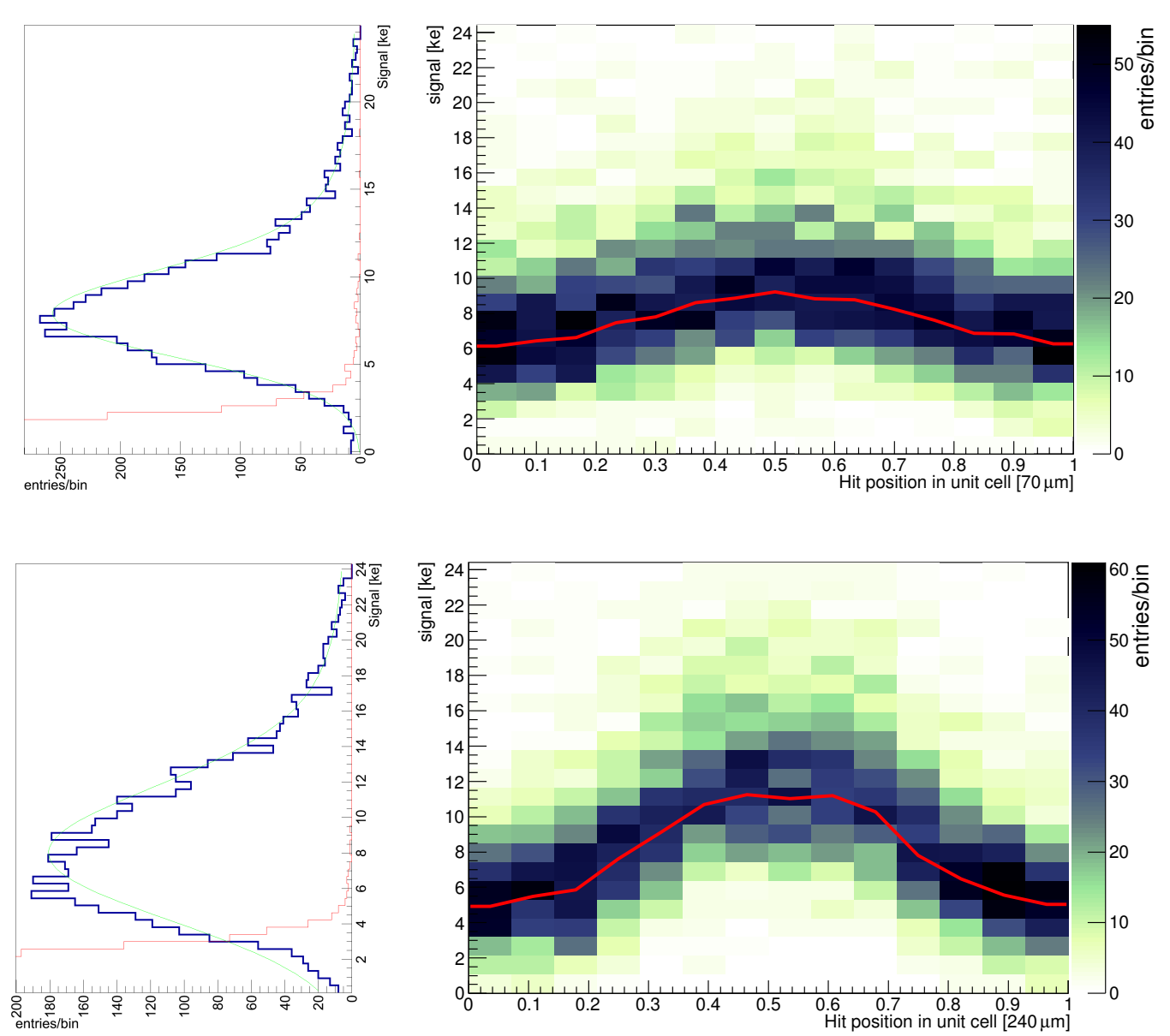

Figure 7: Collected charge (ordinate) of MCZ200Y_05_MSSD_1_p+_8e14_n_5e14 as a function of track impact position (abscissa, strip being at $x=0.5$ ) in the plots at right. The most probable value of individual bins is shown in red. The upper right plot shows region 4 at $400 \mathrm{~V}$, the lower right plot shows region 2 at $600 \mathrm{~V}$. The left plots depict signal histograms of all the clusters; corresponding function fits and noise tails are also shown.

[6] M. J. Kortelainen et al., Off-line calibration and data analysis for the silicon beam telescope on the CERN H2 beam, Nucl. Instr. \& Meth. A, vol. 602 (2009) 600-6

[7] T. Mäenpää et al., Track-induced clustering in position sensitive detector characterization, IEEE Trans. Nucl. Sci. vol. 574 (2010) 2196-9

[8] CMS Collaboration, Physics Technical Design Report Vol. I, CERN-LHCC-2006-001.

[9] M. Petasecca et al., Numerical Simulation of Radiation Damage Effects in p-Type and n-Type FZ Silicon Detectors, Trans. Nucl, Sci, vol. 535 (2006) 2971-6

[10] L. Spiegel et al., Czochralski silicon as a detector material for S-LHC tracker volumes, Nucl. Instr. \& Meth. A, vol. 628 (2011) 242-5

[11] J. Bernardini et al., CCE measurements on heavily irradiated micro-strip sensors Nucl. Instr. \& Meth. $A$, vol. 612 (2010) 478-81 